\title{
Comparative Balancing of Non-Redox and Redox Electrolytic Systems and Its Consequences
}

\author{
Anna Maria Michalowska-Kaczmarczyk ${ }^{1}$, Tadeusz Michalowski ${ }^{2 *}$ \\ ${ }^{1}$ Department of Oncology, The University Hospital in Cracow, Cracow, Poland \\ ${ }^{2}$ Department of Analytical Chemistry, Technical University of Cracow, Cracow, Poland \\ Email: ${ }^{*}$ michalot@o2.pl
}

Received May 31, 2013; revised July 1, 2013; accepted August 15, 2013

Copyright (C) 2013 Anna Maria Michałowska-Kaczmarczyk, Tadeusz Michałowski. This is an open access article distributed under the Creative Commons Attribution License, which permits unrestricted use, distribution, and reproduction in any medium, provided the original work is properly cited.

\begin{abstract}
In this paper, it is proved that linear combination $2 \cdot f(\mathrm{O})-f(\mathrm{H})$ of elemental balances: $f(\mathrm{O})$ for $\mathrm{O}$ and $f(\mathrm{H})$ for $\mathrm{H}$ is linearly independent on charge and elemental/core balances for all redox systems of any degree of complexity; it is the primary form of the Generalized Electron Balance (GEB), $p r-\mathrm{GEB}=2 \cdot f(\mathrm{O})-f(\mathrm{H})$, considered as the Approach II to GEB. The Approach II is equivalent to the Approach I based on the principle of common pool of electrons. Both Approaches are illustrated on an example of titration of acidified $\left(\mathrm{H}_{2} \mathrm{SO}_{4}\right)$ solution of $\mathrm{H}_{2} \mathrm{C}_{2} \mathrm{O}_{4}$ with $\mathrm{KMnO}_{4}$. It is also stated, on an example of titration of the same solution with $\mathrm{NaOH}$, that $2 \cdot f(\mathrm{O})-f(\mathrm{H})$ is a linear combination of charge and elemental/core balances, i.e. it is not an independent balance when related to the non-redox system. These properties of $2 \cdot f(\mathrm{O})-f(\mathrm{H})$ can be extended on redox and non-redox systems, of any degree of complexity, i.e. the linear independency/dependency of $2 \cdot f(\mathrm{O})-f(\mathrm{H})$ on other balances related to a system in question is a criterion distinguishing redox and non-redox systems. The GEB completes the set of (charge and concentration) balances and a set of expressions for independent equilibrium constants needed for modeling the related redox system.
\end{abstract}

Keywords: Electrolytic Systems; Redox Systems; GEB; GATES

\section{Introduction}

Before 1992, the principle of formulation of a complete set of balances related to a redox system was unknown; except $k$ concentration balances and charge balance, the $k$ +2 -th balance, completing the set of equations with $k+2$ variables, was needed. All the trials [1-13] made after 1960 s to find the missing equation were unsuccessful. Those trials were slavishly related to the stoichiometric reaction notations, involving only two pairs of species participating in redox reaction; the species different from those involved in the reaction notation were thus omitted in considerations. What is more, the charge balance and concentration balances for accompanying substances were also omitted. Theoretical considerations were related to virtual cases, not to real, electrolytic redox systems. In conclusion, all authors of those papers did not have any idea on how to resolve correctly the problem in question. The principle of the formulation of the $k+2$-th equation, completing $k+1$ equations composed of $\mathrm{k}$

\footnotetext{
"Corresponding author.
}

concentration balances and charge balance, was devised in 1992 by Michałowski in a totally mature form, known presently as the Approach I to Generalized Electron Balance (GEB). This Approach is based on a card game principle, with electron-active elements as "players", electron-non-active elements as "fans", and electrons as "money" (cash), constituting a common pool of electrons. The common pool of electrons is ascribed to players, whereas the fans' cashes are untouched. The species in the related systems are considered in their natural form, i.e., as hydrates in aqueous media. For the species of $i$-th kind, $X_{i}^{z_{i}} \cdot n_{i} \mathrm{H}_{2} \mathrm{O}$, we apply the notation $X_{i}^{z_{i}}\left(N_{i}, n_{i}\right)$, where $n_{i} \geq 0, N_{i}$ is a number of these entities. The Approach I was formulated by Michałowski within the Generalized Approach to Electrolytic Systems (GATES). The GATES provides a comprehensive, compatible and consistent knowledge on thermodynamics of electrolytic redox and non-redox systems, referred to aqueous and non-aqueous media. The formulation of GATES, with GEB concept referred to redox systems, is denoted briefly as GATES/GEB. The first works on GATES, with the 
Approach I to GEB, were published in 1994 [14-16], and followed by other papers [17-23]. Later on, Michałowski devised the Approach II to GEB.

The Approach II to GEB, presented first in [24] and then in other issues [25-30], is based on a linear combination $2 \cdot f(\mathrm{O})-f(\mathrm{H})$ of the balances: $f(\mathrm{O})$ for oxygen $(\mathrm{O})$ and $f(\mathrm{H})$ for hydrogen $(\mathrm{H})$ related to redox systems; the balance $2 \cdot f(\mathrm{O})-f(\mathrm{H})$ is considered as the primary form of GEB in redox systems $p r-\mathrm{GEB}=2 \cdot f(\mathrm{O})-f(\mathrm{H})$. The pr-GEB is linearly independent on charge balance and other concentration balances in any electrolytic redox system (aqueous and non-aqueous media), whereas in any non-redox system the $2 \cdot f(\mathrm{O})-f(\mathrm{H})$ is dependent on those balances. This property is thus the basis for a distinction between redox and non-redox electrolytic systems. The Approach II is fully equivalent to the Approach I. However, the fundamental advantage of the Approach II (in context with the Approach I) to GEB is that none prior knowledge on oxidation degrees of elements in complex species of definite elemental composition and charge is needed. This property of the Approach II to GEB is advantageous/desired, inter alia, for redox systems where radical and ion-radical species are formed [31-35]. The Approach I can be considered as a "short" version of GEB applicable in the cases where calculation of oxidation degrees of all elements in a system is easy.

The Approach II to GEB can be formulated for static (batch) and dynamic (titration) systems. In any titration, a titrant (T) is added into titrand (solution titrated, D) and a D + T system is thus formed. It is assumed that the D + $\mathrm{T}$ system and the subsystems (D and $\mathrm{T}$ ) are closed (sub) systems, separated from the environment by diathermal walls; any flow of a matter between the environment and the system and its subsystems is not possible. The diathermal walls enable the related process (i.e., titration) to be carried out under isotheral conditions; constancy of temperature is one of the conditions assuring constancy of the related equilibrium constants values. Such titration is an approximation of a real titration.

The GEB is principally formulated for the systems where none nuclear transformations (involved with $\alpha, \beta$, $\beta^{+}$radioactive decay, electron capture, and $\gamma$ radiation) of elements occur, although its extension on such systems is also possible. In this paper, the systems with stable isotopes are considered.

From a mathematical viewpoint, the GEB formulated for any (static or dynamic) redox system is the equation linearly independent on charge and concentration balances. The GEB is fully compatible with these balances related to the system in question. This compatibility, resulting from the "common root" principle, is directly seen from the viewpoint of the Approach II to GEB.

In compliance with the thesis put in the title of this paper, we refer first to the concept of linear dependency or independency of linear algebraic equations, known from elementary algebra [36].

\section{Linear Dependency of Balances}

In algebra, a system of linear equations is a collection of two or more linear equations, involving the same set of variables in all the equations. The set of $\mathrm{m}$ linear equations with $\mathrm{k}$ variables represented by the vector $\boldsymbol{x}=\left[x_{1}, x_{2} \cdots x_{n}\right]^{T}$ has the form $(T$ - transposition sign $)$

$$
\begin{aligned}
& a_{11} x_{1}+a_{12} x_{2}+\cdots+a_{1 n} x_{n}=b_{1} \\
& a_{21} x_{1}+a_{22} x_{2}+\cdots+a_{2 n} x_{n}=b_{2} \\
& \vdots \\
& a_{m 1} x_{1}+a_{m 2} x_{2}+\cdots+a_{m n} x_{n}=b_{m}
\end{aligned}
$$

where $a_{i j}$ are numerical coefficients; some of the $a_{i j}$ values can be equal zero. To facilitate further reasoning, let us consider, for simplicity, the system of $m=3$ equations with $n=2$ variables, $\quad \boldsymbol{x}=\left[x_{1}, x_{2}\right]^{\mathrm{T}}$, i.e.

$$
\begin{gathered}
a_{11} x_{1}+a_{12} x_{2}=b_{1} \\
a_{21} x_{1}+a_{22} x_{2}=b_{2} \\
a_{31} x_{1}+a_{32} x_{2}=b_{3}
\end{gathered}
$$

where (1) and (2) are independent equations, i.e.

$$
\mathrm{D}=a_{11} a_{22}-a_{12} a_{21}
$$

is $\neq 0$. For example, let us take the equations:

$$
\begin{gathered}
x_{1}+x_{2}=3\left(a_{11}=a_{12}=1\right) \\
x_{1}-x_{2}=1\left(a_{21}=1, a_{22}=-1\right) \\
x_{1}-7 x_{2}=-5
\end{gathered}
$$

On the basis of (1a), (2a), from (4) we get

$$
\mathrm{D}=1 \cdot(-1)-1 \cdot 1=-2 \neq 0,
$$

and then $x_{1}=2, x_{2}=1$. Multiplication of (1a) by $-3,(2 \mathrm{a})$ by 4 and addition of the sides of the resulting equations gives (3a), i.e., (3a) is a linear combination of (1a) and (2a). Then (3a) is not an independent equation in this collection of equations, i.e., it depends linearly on (1a) and (2a).

In this paper, we apply more convenient (shorter) procedure to check the dependency of linear equations. This procedure is based on obtaining the identity, equivalent to the statement that $0=0$. For this purpose, we multiply (1a) by 3 , (2a) by -4 and add the related sum to (3a)

$$
\begin{array}{r}
3 x_{1}+3 x_{2}=9 \\
-4 x_{1}+4 x_{2}=-4 \\
x_{1}-7 x_{2}=-5
\end{array}
$$

Adding the Equations: (1b), (2b), (3b), we get the identity, $0=0$ (see also Appendix).

On this simple principle, we prove the linear de- 
pendency of elementary and core $\left(^{\bullet}\right)$ balances relating to non-redox electrolytic systems, of different degree of complexity. On this basis, we put forward a thesis that it is a property of general nature, applicable to non-redox electrolytic systems of any degree of complexity.

\subsection{A Non-Redox System (System A)}

$\mathrm{V} \mathrm{mL}$ of $\mathrm{T}$ containing $\mathrm{C} \mathrm{mol} / \mathrm{L} \mathrm{NaOH}+\mathrm{C}_{2} \mathrm{~mol} / \mathrm{L} \mathrm{CO}_{2}$ is added into $\mathrm{V}_{0} \mathrm{~mL}$ of $\mathrm{D}$ containing $\mathrm{H}_{2} \mathrm{C}_{2} \mathrm{O}_{4}\left(\mathrm{C}_{0}\right)+\mathrm{H}_{2} \mathrm{SO}_{4}$ $\left(\mathrm{C}_{0 \mathrm{z} 1}\right)+\mathrm{C}_{02} \mathrm{~mol} / \mathrm{L} \mathrm{CO} \mathrm{CO}_{2} \cdot \mathrm{V}_{0} \mathrm{~mL}$ of $\mathrm{D}$ is composed of $\mathrm{N}_{10}$ molecules of $\mathrm{H}_{2} \mathrm{C}_{2} \mathrm{O}_{4} \cdot 2 \mathrm{H}_{2} \mathrm{O}+\mathrm{N}_{20}$ molecules of $\mathrm{CO}_{2}+$ $\mathrm{N}_{30}$ molecules of $\mathrm{H}_{2} \mathrm{SO}_{4}+\mathrm{N}_{40}$ molecules of $\mathrm{H}_{2} \mathrm{O}$ and $\mathrm{V}$ $\mathrm{mL}$ of $\mathrm{T}$ is composed of $\mathrm{N}_{50}$ molecules of $\mathrm{NaOH}+\mathrm{N}_{60}$ molecules of $\mathrm{CO}_{2}+\mathrm{N}_{70}$ molecules of $\mathrm{H}_{2} \mathrm{O}$. The following species:

$\mathrm{H}_{2} \mathrm{O}\left(\mathrm{N}_{1}\right) ; \mathrm{H}^{+}\left(\mathrm{N}_{2}, \mathrm{n}_{2}\right), \mathrm{OH}^{-}\left(\mathrm{N}_{3}, \mathrm{n}_{3}\right), \mathrm{HSO}_{4}^{-}\left(\mathrm{N}_{4}, \mathrm{n}_{4}\right)$, $\mathrm{SO}_{4}^{2-}\left(\mathrm{N}_{5}, \mathrm{n}_{5}\right) ; \mathrm{Na}^{+}\left(\mathrm{N}_{6}, \mathrm{n}_{6}\right), \mathrm{H}_{2} \mathrm{C}_{2} \mathrm{O}_{4}\left(\mathrm{~N}_{7}, \mathrm{n}_{7}\right), \mathrm{HC}_{2} \mathrm{O}_{4}^{-}$ $\left(\mathrm{N}_{8}, \mathrm{n}_{8}\right), \mathrm{C}_{2} \mathrm{O}_{4}^{2-}\left(\mathrm{N}_{9}, \mathrm{n}_{9}\right) ; \mathrm{H}_{2} \mathrm{CO}_{3}\left(\mathrm{~N}_{11}, \mathrm{n}_{11}\right), \mathrm{HCO}_{3}^{-}$ $\left(\mathrm{N}_{12}, \mathrm{n}_{12}\right), \mathrm{CO}_{3}^{2-}\left(\mathrm{N}_{13}, \mathrm{n}_{13}\right)$ are formed in the system in question. Note, for example, that $\mathrm{N}_{10}$ molecules of $\mathrm{H}_{2} \mathrm{C}_{2} \mathrm{O}_{4} \cdot 2 \mathrm{H}_{2} \mathrm{O}$ introduce $6 \mathrm{~N}_{10}$ atoms of $\mathrm{H}, 6 \mathrm{~N}_{10}$ atoms of $\mathrm{O}$ and $2 \mathrm{~N}_{10}$ atoms of $\mathrm{C} ; \mathrm{N}_{8}$ ions of $\mathrm{HC}_{2} \mathrm{O}_{4}^{-} \cdot \mathrm{n}_{8} \mathrm{H}_{2} \mathrm{O}$ contain $\mathrm{N}_{8}\left(1+2 \mathrm{n}_{8}\right)$ atoms of $\mathrm{H}, \mathrm{N}_{8}\left(4+\mathrm{n}_{8}\right)$ atoms of $\mathrm{O}$ and $2 \mathrm{~N}_{8}$ atoms of $\mathrm{C}$. On this basis, one can formulate the balances:

$f(\mathrm{H})$

$$
\begin{aligned}
& 2 \mathrm{~N}_{1}+\mathrm{N}_{2}\left(1+2 \mathrm{n}_{2}\right)+\mathrm{N}_{3}\left(1+2 \mathrm{n}_{3}\right)+\mathrm{N}_{4}\left(1+2 \mathrm{n}_{4}\right) \\
& +2 \mathrm{~N}_{5} \mathrm{n}_{5}+2 \mathrm{~N}_{6} \mathrm{n}_{6}+\mathrm{N}_{7}\left(2+2 \mathrm{n}_{7}\right)+\mathrm{N}_{8}\left(1+2 \mathrm{n}_{8}\right) \\
& +2 \mathrm{~N}_{9} \mathrm{n}_{9}+\mathrm{N}_{11}\left(2+2 \mathrm{n}_{11}\right)+\mathrm{N}_{12}\left(1+2 \mathrm{n}_{12}\right)+2 \mathrm{~N}_{13} \mathrm{n}_{13} \\
& =6 \mathrm{~N}_{10}+2 \mathrm{~N}_{30}+2 \mathrm{~N}_{40}+\mathrm{N}_{50}+2 \mathrm{~N}_{70}
\end{aligned}
$$

$f(\mathrm{O})$

$$
\begin{aligned}
& \mathrm{N}_{1}+\mathrm{N}_{2} \mathrm{n}_{2}+\mathrm{N}_{3}\left(1+\mathrm{n}_{3}\right)+\mathrm{N}_{4}\left(4+\mathrm{n}_{4}\right)+\mathrm{N}_{5}\left(4+\mathrm{n}_{5}\right) \\
& +\mathrm{N}_{6} \mathrm{n}_{6}+\mathrm{N}_{7}\left(4+\mathrm{n}_{7}\right)+\mathrm{N}_{8}\left(4+\mathrm{n}_{8}\right)+\mathrm{N}_{9}\left(4+\mathrm{n}_{9}\right) \\
& +\mathrm{N}_{11}\left(3+\mathrm{n}_{11}\right)+\mathrm{N}_{12}\left(3+\mathrm{n}_{12}\right)+\mathrm{N}_{13}\left(3+\mathrm{n}_{13}\right) \\
& =6 \mathrm{~N}_{10}+2 \mathrm{~N}_{20}+4 \mathrm{~N}_{30}+\mathrm{N}_{40}+\mathrm{N}_{50}+2 \mathrm{~N}_{60}+\mathrm{N}_{70} \\
& 2 f(\mathrm{O})-f(\mathrm{H}) \\
& -\mathrm{N}_{2}+\mathrm{N}_{3}+7 \mathrm{~N}_{4}+8 \mathrm{~N}_{5}+6 \mathrm{~N}_{7}+7 \mathrm{~N}_{8}+8 \mathrm{~N}_{9}+4 \mathrm{~N}_{11} \\
& +5 \mathrm{~N}_{12}+6 \mathrm{~N}_{13}=6 \mathrm{~N}_{10}+4 \mathrm{~N}_{20}+6 \mathrm{~N}_{30}+\mathrm{N}_{50}+4 \mathrm{~N}_{60}
\end{aligned}
$$

Addition of (6) to charge balance (7), $6 \cdot f\left(\mathrm{SO}_{4}\right)(8)$, $6 \cdot f\left(\mathrm{C}_{2} \mathrm{O}_{4}\right)(9), 4 \cdot f\left(\mathrm{CO}_{3}\right)(10)$ and $f(\mathrm{Na})(11)$

$$
\mathrm{N}_{2}-\mathrm{N}_{3}-\mathrm{N}_{4}-2 \mathrm{~N}_{5}+\mathrm{N}_{6}-\mathrm{N}_{8}-2 \mathrm{~N}_{9}-\mathrm{N}_{12}-2 \mathrm{~N}_{13}=0
$$

\footnotetext{
'The term "core", when referred to the species in electrolytic systems, means a common group of elements of the same composition and structure; e.g., $\mathrm{C}_{2} \mathrm{O}_{4}$ is a common core for different oxalate clusters: $\mathrm{H}_{2} \mathrm{C}_{2} \mathrm{O}_{4}, \mathrm{HC}_{2} \mathrm{O}_{4}^{-}$and $\mathrm{C}_{2} \mathrm{O}_{4}^{2-} ; \mathrm{SO}_{4}$ is a common core for different sulfate clusters: $\mathrm{HSO}_{4}^{-}, \mathrm{SO}_{4}^{2-}$ and $\mathrm{MnSO}_{4}$.
}

$$
\begin{gathered}
6 \mathrm{~N}_{30}=6 \mathrm{~N}_{4}+6 \mathrm{~N}_{5} \\
6 \mathrm{~N}_{10}=6 \mathrm{~N}_{7}+6 \mathrm{~N}_{8}+6 \mathrm{~N}_{9} \\
4 \mathrm{~N}_{20}+4 \mathrm{~N}_{60}=4 \mathrm{~N}_{11}+4 \mathrm{~N}_{12}+4 \mathrm{~N}_{13} \\
\mathrm{~N}_{50}=\mathrm{N}_{6}
\end{gathered}
$$

gives the identity, $0=0$

\subsection{A Redox System (System B)}

$\mathrm{V} \mathrm{mL}$ of $\mathrm{T}$ containing $\mathrm{C} \mathrm{mol} / \mathrm{L} \mathrm{KMnO}_{4}+\mathrm{C}_{2} \mathrm{~mol} / \mathrm{L} \mathrm{CO}_{2}$ is added into $\mathrm{V}_{0} \mathrm{~mL}$ of $\mathrm{D}$ containing $\mathrm{H}_{2} \mathrm{C}_{2} \mathrm{O}_{4}\left(\mathrm{C}_{0}\right)+$ $\mathrm{H}_{2} \mathrm{SO}_{4}\left(\mathrm{C}_{01}\right)+\mathrm{C}_{02} \mathrm{~mol} / \mathrm{L} \mathrm{CO}_{2} . \mathrm{V}_{0} \mathrm{~mL}$ of $\mathrm{D}$ is composed of $\mathrm{N}_{10}$ molecules of $\mathrm{H}_{2} \mathrm{C}_{2} \mathrm{O}_{4} \cdot 2 \mathrm{H}_{2} \mathrm{O}+\mathrm{N}_{20}$ molecules of $\mathrm{CO}_{2}+\mathrm{N}_{30}$ molecules of $\mathrm{H}_{2} \mathrm{SO}_{4}+\mathrm{N}_{40}$ molecules of $\mathrm{H}_{2} \mathrm{O}$ and $\mathrm{V}$ mL of $\mathrm{T}$ is composed of $\mathrm{N}_{50}$ molecules of $\mathrm{KMnO}_{4}$ $+\mathrm{N}_{60}$ molecules of $\mathrm{CO}_{2}+\mathrm{N}_{70}$ molecules of $\mathrm{H}_{2} \mathrm{O}$. The following species:

$\mathrm{H}_{2} \mathrm{O}\left(\mathrm{N}_{1}\right) ; \mathrm{H}^{+}\left(\mathrm{N}_{2}, \mathrm{n}_{2}\right), \mathrm{OH}^{-}\left(\mathrm{N}_{3}, \mathrm{n}_{3}\right), \mathrm{HSO}_{4}^{-}\left(\mathrm{N}_{4}, \mathrm{n}_{4}\right)$, $\mathrm{SO}_{4}^{2-}\left(\mathrm{N}_{5}, \mathrm{n}_{5}\right) ; \mathrm{K}^{+}\left(\mathrm{N}_{6}, \mathrm{n}_{6}\right), \mathrm{H}_{2} \mathrm{C}_{2} \mathrm{O}_{4}\left(\mathrm{~N}_{7}, \mathrm{n}_{7}\right), \mathrm{HC}_{2} \mathrm{O}_{4}^{-}$ $\left(\mathrm{N}_{8}, \mathrm{n}_{8}\right), \mathrm{C}_{2} \mathrm{O}_{4}^{2-}\left(\mathrm{N}_{9}, \mathrm{n}_{9}\right) ; \mathrm{H}_{2} \mathrm{CO}_{3}\left(\mathrm{~N}_{11}, \mathrm{n}_{11}\right), \mathrm{HCO}_{3}^{-}$ $\left(\mathrm{N}_{12}, \mathrm{n}_{12}\right), \mathrm{CO}_{3}^{2-}\left(\mathrm{N}_{13}, \mathrm{n}_{13}\right) ; \mathrm{MnO}_{4}^{-}\left(\mathrm{N}_{14}, \mathrm{n}_{14}\right), \mathrm{MnO}_{4}^{2-}$ $\left(\mathrm{N}_{15}, \mathrm{n}_{15}\right), \mathrm{Mn}^{3+}\left(\mathrm{N}_{16}, \mathrm{n}_{16}\right), \mathrm{MnOH}^{2+}\left(\mathrm{N}_{17}, \mathrm{n}_{17}\right), \mathrm{Mn}^{2+}\left(\mathrm{N}_{18}\right.$, $\left.\mathrm{n}_{18}\right), \mathrm{MnOH}^{+}\left(\mathrm{N}_{19}, \mathrm{n}_{19}\right), \mathrm{MnSO}_{4}\left(\mathrm{~N}_{21}, \mathrm{n}_{21}\right)$ are formed in the system in question. Before addition of the first portion of the titrant $(\mathrm{T})$, the solution is heated up to ca. $80^{\circ} \mathrm{C}$; it is a metastable system at room temperature [26,28].

This system will be considered from the viewpoints of Approaches I and II to GEB. In the Approach I, Mn and $\mathrm{C}$ are considered as players whose electrons are balanced. The players are involved with fans in hydrates or other complexes.

\subsubsection{Approach I to GEB}

Denoting atomic numbers of $\mathrm{Mn}$ and $\mathrm{C}$ by $\mathrm{Z}_{\mathrm{Mn}}=25$ and $\mathrm{Z}_{\mathrm{C}}=6$, we state that $\mathrm{N}_{10}$ molecules of $\mathrm{KMnO}_{4}$ introduce $\mathrm{N}_{50}\left(Z_{\mathrm{Mn}}-7\right)$ electrons, whereas the carbon components introduce $2\left(Z_{C}-3\right) N_{10}+\left(Z_{C}-4\right) N_{20}+\left(Z_{C}-4\right) N_{60}$ electrons to the common pool. In the resulting $\mathrm{D}+\mathrm{T}$ system (solution), $\mathrm{N}_{7}$ entities $\mathrm{H}_{2} \mathrm{C}_{2} \mathrm{O}_{4} \cdot \mathrm{n}_{7} \mathrm{H}_{2} \mathrm{O}$ involve $2\left(\mathrm{Z}_{\mathrm{C}}-3\right)$ $\mathrm{N}_{7}$ electrons, $\mathrm{N}_{8}$ entities $\mathrm{HC}_{2} \mathrm{O}_{4}^{2-} \cdot \mathrm{n}_{8} \mathrm{H}_{2} \mathrm{O}$ involve $2\left(\mathrm{Z}_{\mathrm{C}}-\right.$ 3) $\mathrm{N}_{8}$ electrons... $\mathrm{N}_{13}$ entities $\mathrm{CO}_{3}^{2-} \cdot \mathrm{n}_{8} \mathrm{H}_{2} \mathrm{O}$ involve $\left(\mathrm{Z}_{\mathrm{C}}\right.$ - 4) $\mathrm{N}_{13}$ electrons, $\mathrm{N}_{14}$ entities $\mathrm{MnO}_{4}^{-} \cdot \mathrm{n}_{14} \mathrm{H}_{2} \mathrm{O}$ involve $\left(\mathrm{Z}_{\mathrm{Mn}}-7\right) \mathrm{N}_{14}$ electrons, ... $\mathrm{N}_{21}$ entities $\mathrm{MnSO}_{4} \cdot \mathrm{n}_{21} \mathrm{H}_{2} \mathrm{O}$ involve $\left(Z_{\mathrm{Mn}}-2\right) \mathrm{N}_{21}$ electrons. Then the following balance is valid

$$
\begin{aligned}
& 2\left(\mathrm{Z}_{\mathrm{C}}-3\right)\left(\mathrm{N}_{7}+\mathrm{N}_{8}+\mathrm{N}_{9}\right)+\left(\mathrm{Z}_{\mathrm{C}}-4\right)\left(\mathrm{N}_{11}+\mathrm{N}_{12}+\mathrm{N}_{13}\right) \\
& +\left(\mathrm{Z}_{\mathrm{Mn}}-7\right) N_{14}+\left(\mathrm{Z}_{\mathrm{Mn}}-6\right) N_{15}+\left(\mathrm{Z}_{\mathrm{Mn}}-3\right)\left(\mathrm{N}_{16}+\mathrm{N}_{17}\right) \\
& +\left(Z_{M n}-2\right)\left(\mathrm{N}_{18}+\mathrm{N}_{19}+\mathrm{N}_{21}\right)=2\left(Z_{C}-3\right) \mathrm{N}_{10} \\
& +\left(Z_{C}-4\right)\left(\mathrm{N}_{20}+\mathrm{N}_{60}\right)+\left(Z_{M n}-7\right) \mathrm{N}_{50}
\end{aligned}
$$

Applying the relations: 


$$
\begin{aligned}
& {\left[\mathrm{X}_{\mathrm{i}}^{\mathrm{Zi}}\right]\left(\mathrm{V}_{0}+\mathrm{V}\right)=10^{3} \cdot \mathrm{N}_{\mathrm{i}} / \mathrm{N}_{\mathrm{A}}, \mathrm{C}_{0} \mathrm{~V}_{0}=10^{3} \cdot \mathrm{N}_{10} / \mathrm{N}_{\mathrm{A}}} \\
& \mathrm{CV}=10^{3} \cdot \mathrm{N}_{50} / \mathrm{N}_{\mathrm{A}}, \mathrm{C}_{01} \mathrm{~V}_{0}=10^{3} \cdot \mathrm{N}_{20} / \mathrm{N}_{\mathrm{A}} \\
& \mathrm{C} \mathrm{V}_{1}=10^{3} \cdot \mathrm{N}_{60} / \mathrm{N}_{\mathrm{A}}
\end{aligned}
$$

in (12), we get the equation

$$
\begin{aligned}
& 2\left(\mathrm{Z}_{\mathrm{C}}-3\right)\left(\left[\mathrm{H}_{2} \mathrm{C}_{2} \mathrm{O}_{4}\right]+\left[\mathrm{HC}_{2} \mathrm{O}_{4}^{-}\right]+\left[\mathrm{C}_{2} \mathrm{O}_{4}^{2-}\right]\right) \\
& +\left(\mathrm{Z}_{\mathrm{C}}-4\right)\left(\left[\mathrm{H}_{2} \mathrm{CO}_{3}\right]+\left[\mathrm{HCO}_{3}^{-}\right]+\left[\mathrm{CO}_{3}^{2-}\right]\right) \\
& +\left(\mathrm{Z}_{\mathrm{Mn}}-7\right)\left[\mathrm{MnO}_{4}^{-}\right]+\left(\mathrm{Z}_{\mathrm{Mn}}-6\right)\left[\mathrm{MnO}_{4}^{2-}\right] \\
& +\left(\mathrm{Z}_{\mathrm{Mn}}-3\right)\left(\left[\mathrm{Mn}^{3+}\right]+\left[\mathrm{MnOH}^{2+}\right]\right) \\
& +\left(\mathrm{Z}_{\mathrm{Mn}}-2\right)\left(\left[\mathrm{Mn}^{2+}\right]+\left[\mathrm{MnOH}^{+}\right]+\left[\mathrm{MnSO}_{4}\right]\right) \\
& =\left(2\left(\mathrm{Z}_{\mathrm{C}}-3\right) \mathrm{C}_{0} \mathrm{~V}_{0}+\left(\mathrm{Z}_{\mathrm{C}}-4\right)\left(\mathrm{C}_{02} \mathrm{~V}_{0}+\mathrm{C}_{2} \mathrm{~V}\right)\right. \\
& \left.+\left(\mathrm{Z}_{\mathrm{Mn}}-7\right) \mathrm{CV}\right) /\left(\mathrm{V}_{0}+\mathrm{V}\right)
\end{aligned}
$$

\subsubsection{Approach II to GEB}

We formulate the balances:

$f(\mathrm{H})$

$$
\begin{aligned}
& 2 \mathrm{~N}_{1}+\mathrm{N}_{2}\left(1+2 \mathrm{n}_{2}\right)+\mathrm{N}_{3}\left(1+2 \mathrm{n}_{3}\right)+\mathrm{N}_{4}\left(1+2 \mathrm{n}_{4}\right) \\
& +2 \mathrm{~N}_{5} \mathrm{n}_{5}+2 \mathrm{~N}_{6} \mathrm{n}_{6}+\mathrm{N}_{7}\left(2+2 \mathrm{n}_{7}\right)+\mathrm{N}_{8}\left(1+2 \mathrm{n}_{8}\right) \\
& +2 \mathrm{~N}_{9} \mathrm{n}_{9}+\mathrm{N}_{11}\left(2+2 \mathrm{n}_{11}\right)+\mathrm{N}_{12}\left(1+2 \mathrm{n}_{12}\right)+2 \mathrm{~N}_{13} \mathrm{n}_{13} \\
& +2 \mathrm{~N}_{14} \mathrm{n}_{14}+2 \mathrm{~N}_{15} \mathrm{n}_{15}+2 \mathrm{~N}_{16} \mathrm{n}_{16}+\mathrm{N}_{17}\left(1+2 \mathrm{n}_{17}\right)+2 \mathrm{~N}_{18} \mathrm{n}_{18} \\
& +\mathrm{N}_{19}\left(1+2 \mathrm{n}_{19}\right)+2 \mathrm{~N}_{21} \mathrm{n}_{21}=6 \mathrm{~N}_{10}+2 \mathrm{~N}_{30}+2 \mathrm{~N}_{40}+2 \mathrm{~N}_{70}
\end{aligned}
$$

$f(\mathrm{O})$

$$
\begin{aligned}
& \mathrm{N}_{1}+\mathrm{N}_{2} \mathrm{n}_{2}+\mathrm{N}_{3}\left(1+\mathrm{n}_{3}\right)+\mathrm{N}_{4}\left(4+\mathrm{n}_{4}\right)+\mathrm{N}_{5}\left(4+\mathrm{n}_{5}\right) \\
& +\mathrm{N}_{6} \mathrm{n}_{6}+\mathrm{N}_{7}\left(4+\mathrm{n}_{7}\right)+\mathrm{N}_{8}\left(4+\mathrm{n}_{8}\right)+\mathrm{N}_{9}\left(4+\mathrm{n}_{9}\right) \\
& +\mathrm{N}_{11}\left(3+\mathrm{n}_{11}\right)+\mathrm{N}_{12}\left(3+\mathrm{n}_{12}\right)+\mathrm{N}_{13}\left(3+\mathrm{n}_{13}\right) \\
& +\mathrm{N}_{14}\left(4+\mathrm{n}_{14}\right)+\mathrm{N}_{15}\left(4+\mathrm{n}_{15}\right)+\mathrm{N}_{16} \mathrm{n}_{16}+\mathrm{N}_{17}\left(1+\mathrm{n}_{17}\right) \\
& +\mathrm{N}_{18} \mathrm{n}_{18}+\mathrm{N}_{19}\left(1+\mathrm{n}_{19}\right)+\mathrm{N}_{21}\left(4+\mathrm{n}_{21}\right) \\
& =6 \mathrm{~N}_{10}+2 \mathrm{~N}_{20}+4 \mathrm{~N}_{30}+\mathrm{N}_{40}+4 \mathrm{~N}_{50}+2 \mathrm{~N}_{60}+\mathrm{N}_{70}
\end{aligned}
$$

$$
2 f(\mathrm{O})-f(\mathrm{H})=p \mathrm{r}-\mathrm{GEB}
$$$$
-\mathrm{N}_{2}+\mathrm{N}_{3}+7 \mathrm{~N}_{4}+8 \mathrm{~N}_{5}+6 \mathrm{~N}_{7}+7 \mathrm{~N}_{8}+8 \mathrm{~N}_{9}+4 \mathrm{~N}_{11}
$$$$
+5 \mathrm{~N}_{12}+6 \mathrm{~N}_{13}+8 \mathrm{~N}_{14}+8 \mathrm{~N}_{15}+\mathrm{N}_{17}+\mathrm{N}_{19}+8 \mathrm{~N}_{21}
$$$$
=6 \mathrm{~N}_{10}+4 \mathrm{~N}_{20}+6 \mathrm{~N}_{30}+8 \mathrm{~N}_{50}+4 \mathrm{~N}_{60}
$$

Addition of (17) to charge balance (18), $6 \cdot f\left(\mathrm{SO}_{4}\right)(19)$ and $f(\mathrm{~K})(20)$

$$
\begin{gathered}
\mathrm{N}_{2}-\mathrm{N}_{3}-\mathrm{N}_{4}-2 \mathrm{~N}_{5}+\mathrm{N}_{6}-\mathrm{N}_{8}-2 \mathrm{~N}_{9}-\mathrm{N}_{12}-2 \mathrm{~N}_{13} \\
-\mathrm{N}_{14}-2 \mathrm{~N}_{15}+3 \mathrm{~N}_{16}+2 \mathrm{~N}_{17}+2 \mathrm{~N}_{18}+\mathrm{N}_{19}=0 \\
6 \mathrm{~N}_{30}=6 \mathrm{~N}_{4}+6 \mathrm{~N}_{5}+6 \mathrm{~N}_{21}
\end{gathered}
$$

$$
\mathrm{N}_{50}=\mathrm{N}_{6}
$$

gives

$$
\begin{aligned}
& 6\left(\mathrm{~N}_{7}+\mathrm{N}_{8}+\mathrm{N}_{9}\right)+4\left(\mathrm{~N}_{11}+\mathrm{N}_{12}+\mathrm{N}_{13}\right) \\
& +7 \mathrm{~N}_{14}+6 \mathrm{~N}_{15}+3\left(\mathrm{~N}_{16}+\mathrm{N}_{17}\right)+2\left(\mathrm{~N}_{18}+\mathrm{N}_{19}+\mathrm{N}_{21}\right) \\
& =6 \mathrm{~N}_{10}+4\left(\mathrm{~N}_{20}+\mathrm{N}_{60}\right)+7 \mathrm{~N}_{50}
\end{aligned}
$$

Subtraction of (21) from $Z_{\mathrm{Mn}} \cdot f(\mathrm{Mn})+\mathrm{Z}_{\mathrm{C}} \cdot f(\mathrm{C})$, i.e.

$$
\begin{aligned}
& Z_{\mathrm{C}} \cdot\left(2 \mathrm{~N}_{7}+2 \mathrm{~N}_{8}+2 \mathrm{~N}_{9}+\mathrm{N}_{11}+\mathrm{N}_{12}+\mathrm{N}_{13}\right)+\mathrm{Z}_{\mathrm{Mn}} \cdot \mathrm{N}_{14} \\
& +\mathrm{Z}_{\mathrm{Mn}} \cdot \mathrm{N}_{15}+\mathrm{Z}_{\mathrm{Mn}} \cdot\left(\mathrm{N}_{16}+\mathrm{N}_{17}\right)+\mathrm{Z}_{\mathrm{Mn}} \cdot\left(\mathrm{N}_{18}+\mathrm{N}_{19}+\mathrm{N}_{21}\right) \\
& =\mathrm{Z}_{\mathrm{C}} \cdot 2 \mathrm{~N}_{10}+\mathrm{Z}_{\mathrm{C}} \cdot\left(\mathrm{N}_{20}+\mathrm{N}_{60}\right)+\mathrm{Z}_{\mathrm{Mn}} \cdot \mathrm{N}_{50}
\end{aligned}
$$

gives

$$
\begin{aligned}
& \left(\mathrm{Z}_{\mathrm{C}}-3\right)\left(2 \mathrm{~N}_{7}+2 \mathrm{~N}_{8}+2 \mathrm{~N}_{9}\right)+\left(\mathrm{Z}_{\mathrm{C}}-4\right)\left(\mathrm{N}_{11}+\mathrm{N}_{12}+\mathrm{N}_{13}\right) \\
& +\left(\mathrm{Z}_{\mathrm{Mn}}-7\right) \mathrm{N}_{14}+\left(\mathrm{Z}_{\mathrm{Mn}}-6\right) \mathrm{N}_{15}+\left(\mathrm{Z}_{\mathrm{Mn}}-3\right)\left(\mathrm{N}_{16}+\mathrm{N}_{17}\right) \\
& +\left(\mathrm{Z}_{\mathrm{Mn}}-2\right)\left(\mathrm{N}_{18}+\mathrm{N}_{19}+\mathrm{N}_{21}\right)=\left(\mathrm{Z}_{\mathrm{C}}-3\right) \cdot 2 \mathrm{~N}_{10} \\
& +\left(\mathrm{Z}_{\mathrm{C}}-4\right)\left(\mathrm{N}_{20}+\mathrm{N}_{60}\right)+\left(\mathrm{Z}_{\mathrm{Mn}}-7\right) \mathrm{N}_{50}
\end{aligned}
$$

Introducing the relations (13) to (22), we get the equation identical with (14); it proves the equivalency of the Approaches I and II to GEB. Equation (14) is considered as GEB for the related system. However, the equation

$$
\begin{aligned}
&-\left[\mathrm{H}^{+}\right]+\left[\mathrm{OH}^{-}\right]+7\left[\mathrm{HSO}_{4}^{-}\right]+8\left[\mathrm{SO}_{4}^{2-}\right]+6\left[\mathrm{H}_{2} \mathrm{C}_{2} \mathrm{O}_{4}\right] \\
&+7\left[\mathrm{HC}_{2} \mathrm{O}_{4}^{-}\right]+8\left[\mathrm{C}_{2} \mathrm{O}_{4}^{2-}\right]+4\left[\mathrm{H}_{2} \mathrm{CO}_{3}\right]+5\left[\mathrm{HCO}_{3}^{-}\right] \\
&+6\left[\mathrm{CO}_{3}^{2-}\right]+8\left[\mathrm{MnO}_{4}^{-}\right]+8\left[\mathrm{MnO}_{4}^{2-}\right]+\left[\mathrm{MnOH}^{2+}\right] \\
&+\left[\mathrm{MnOH}^{+}\right]+8\left[\mathrm{MnSO}_{4}\right]=\left(6 \mathrm{C}_{0} \mathrm{~V}_{0}+4\left(\mathrm{C}_{02} \mathrm{~V}_{0}+\mathrm{C}_{2} \mathrm{~V}\right)\right. \\
&\left.+6 \mathrm{C}_{01} \mathrm{~V}_{0}+8 \mathrm{CV}\right) /\left(\mathrm{V}_{0}+\mathrm{V}\right)
\end{aligned}
$$

obtained after application of the relations (13) and $\mathrm{C}_{01} \mathrm{~V}_{0}=10^{3} \cdot \mathrm{N}_{30} / \mathrm{N}_{\mathrm{A}}$ in (17) and any other combination of (17) with other (charge and/or elemental/core) balances provides an equivalent form of GEB. For example, subtraction of $\left(Z_{C}-4\right) \cdot f(C)+\left(Z_{M n}-2\right) \cdot f(M n)$ from (12) and application of (13) gives the simplest (in terms of the number of components) form of GEB

$$
\begin{aligned}
& 2\left(\left[\mathrm{H}_{2} \mathrm{C}_{2} \mathrm{O}_{4}\right]+\left[\mathrm{HC}_{2} \mathrm{O}_{4}^{-}\right]+\left[\mathrm{C}_{2} \mathrm{O}_{4}^{2-}\right]\right) \\
& -\left(5\left[\mathrm{MnO}_{4}^{-}\right]+4\left[\mathrm{MnO}_{4}^{2-}\right]+\left[\mathrm{Mn}^{3+}\right]+\left[\mathrm{MnOH}^{2+}\right]\right) \\
& =\left(2 \mathrm{C}_{0} \mathrm{~V}_{0}-5 \mathrm{CV}\right) /\left(\mathrm{V}_{0}+\mathrm{V}\right)
\end{aligned}
$$

which is one of the equivalent forms of GEB, referring to the system in question. As we see, application to the redox system of the same procedure, i.e., linear combination of the corresponding set of equations, does not led to the identity, but a new balance, independent from charge and concentration balances, is obtained. 


\section{Completing the Set of Balances}

The GEB, expressed e.g. by Equation (24), is completed by charge (see Equation (18))

$$
\begin{aligned}
& {\left[\mathrm{H}^{+}\right]-\left[\mathrm{OH}^{-}\right]-\left[\mathrm{HSO}_{4}^{-}\right]-2\left[\mathrm{SO}_{4}^{2-}\right]+\left[\mathrm{K}^{+}\right]} \\
& -\left[\mathrm{HC}_{2} \mathrm{O}_{4}^{-}\right]-2\left[\mathrm{C}_{2} \mathrm{O}_{4}^{2-}\right]-\left[\mathrm{HCO}_{3}^{-}\right]-2\left[\mathrm{CO}_{3}^{2-}\right] \\
& -\left[\mathrm{MnO}_{4}^{-}\right]-2\left[\mathrm{MnO}_{4}^{2-}\right]+3\left[\mathrm{Mn}^{3+}\right]+2\left[\mathrm{MnOH}^{2+}\right] \\
& +2\left[\mathrm{Mn}^{2+}\right]+\left[\mathrm{MnOH}^{+}\right]=0
\end{aligned}
$$

and concentration balances:

$f(\mathrm{C})$

$$
\begin{aligned}
& 2\left[\mathrm{H}_{2} \mathrm{C}_{2} \mathrm{O}_{4}\right]+2\left[\mathrm{HC}_{2} \mathrm{O}_{4}^{-}\right]+2\left[\mathrm{C}_{2} \mathrm{O}_{4}^{2-}\right]+\left[\mathrm{H}_{2} \mathrm{CO}_{3}\right] \\
& +\left[\mathrm{HCO}_{3}^{-}\right]+\left[\mathrm{CO}_{3}^{2-}\right]=\left(2 \mathrm{C}_{0} \mathrm{~V}_{0}+\mathrm{C}_{02} \mathrm{~V}_{0}+\mathrm{C}_{2} \mathrm{~V}\right) /\left(\mathrm{V}_{0}+\mathrm{V}\right)
\end{aligned}
$$

$f(\mathrm{Mn})$

$$
\begin{aligned}
& {\left[\mathrm{MnO}_{4}^{-}\right]+\left[\mathrm{MnO}_{4}^{2-}\right]+\left[\mathrm{Mn}^{3+}\right]+\left[\mathrm{MnOH}^{2+}\right]+\left[\mathrm{Mn}^{2+}\right]} \\
& +\left[\mathrm{MnOH}^{+}\right]+\left[\mathrm{MnSO}_{4}\right]=\mathrm{CV} /\left(\mathrm{V}_{0}+\mathrm{V}\right)
\end{aligned}
$$

$$
\begin{aligned}
& f(\mathrm{~S})\left(\text { or } f\left(\mathrm{SO}_{4}\right)\right) \\
& {\left[\mathrm{HSO}_{4}^{-}\right]+\left[\mathrm{SO}_{4}^{2-}\right]+\left[\mathrm{MnSO}_{4}\right]=\mathrm{C}_{01} \mathrm{~V}_{0} /\left(\mathrm{V}_{0}+\mathrm{V}\right)}
\end{aligned}
$$

The relation $\left[\mathrm{K}^{+}\right]=\mathrm{CV} /\left(\mathrm{V}_{0}+\mathrm{V}\right)$ (see Equation (20)) is not considered as a concentration balance; at defined $\mathrm{C}, \mathrm{V}$ and $\mathrm{V}_{0}$ values, it enters as a number in the charge balance (25).

It should be noticed that the species with different cores, namely $\mathrm{C}_{2} \mathrm{O}_{4}$ and $\mathrm{CO}_{3}$, are involved in the same balance (26). It results from the fact that oxalate species are transformed in the System B into carbonate species, i.e., a change in the core occurs. Such a transformation does not occur in the System A, where the balances for oxalates and carbonates can be written as separate equations.

\section{Completing the Set of Equilibrium Constants}

The set of (GEB, charge and concentration) balances is completed by interrelations between concentrations of the species entering these balances. These interrelations are expressed by a set of expressions for independent equilibrium constants. These equilibrium constants, obtained from tables of equilibrium constants [37-39] values, are involved with non-redox:

$$
\begin{aligned}
\mathrm{H}_{2} \mathrm{O}= & \mathrm{H}^{+}+\mathrm{OH}^{-}\left(\mathrm{pK}_{\mathrm{w}}=14\right),\left[\mathrm{OH}^{-}\right]=10^{\mathrm{pH}-14} \\
& \mathrm{H}_{2} \mathrm{C}_{2} \mathrm{O}_{4}=\mathrm{H}^{+}+\mathrm{HC}_{2} \mathrm{O}_{4}^{-}\left(\mathrm{pK}_{1}=1.25\right) \\
& {\left[\mathrm{HC}_{2} \mathrm{O}_{4}^{-}\right]=10^{\mathrm{pH}-1.25} \cdot\left[\mathrm{H}_{2} \mathrm{C}_{2} \mathrm{O}_{4}\right] }
\end{aligned}
$$

$$
\begin{gathered}
\mathrm{HC}_{2} \mathrm{O}_{4}^{-}=\mathrm{H}^{+}+\mathrm{C}_{2} \mathrm{O}_{4}^{2-}\left(\mathrm{pK}_{2}=4.27\right) \\
{\left[\mathrm{C}_{2} \mathrm{O}_{4}^{2-}\right]=10^{\mathrm{pH}-4.27} \cdot\left[\mathrm{HC}_{2} \mathrm{O}_{4}^{-}\right]} \\
\mathrm{H}_{2} \mathrm{CO}_{3}=\mathrm{H}^{+}+\mathrm{HCO}_{3}^{-}\left(\mathrm{pK}_{1}=6.3\right) \\
{\left[\mathrm{HCO}_{3}^{-}\right]=10^{\mathrm{pH}-6.3} \cdot\left[\mathrm{H}_{2} \mathrm{CO}_{3}\right]} \\
\mathrm{HCO}_{3}^{-}=\mathrm{H}^{+}+\mathrm{CO}_{3}^{2-}\left(\mathrm{pK}_{2}=10.1\right) \\
{\left[\mathrm{CO}_{3}^{2-}\right]=10^{\mathrm{pH}-10.1} \cdot\left[\mathrm{HCO}_{3}^{-}\right]} \\
\mathrm{HSO}_{4}^{-}=\mathrm{H}^{+}+\mathrm{SO}_{4}^{2-}\left(\mathrm{pK}_{2}=1.8\right) \\
{\left[\mathrm{SO}_{4}^{2-}\right]=10^{\mathrm{pH}-1.8} \cdot\left[\mathrm{HSO}_{4}^{-}\right]} \\
\mathrm{Mn}^{3+}+\mathrm{OH}^{-}=\mathrm{MnOH}^{2+}\left(\log \mathrm{K}_{1}^{\mathrm{OH}}=14.2\right) \\
{\left[\mathrm{MnOH}^{2+}\right]=10^{\mathrm{pH}+0.2} \cdot\left[\mathrm{Mn}^{3+}\right]} \\
\mathrm{Mn}^{2+}+\mathrm{OH}^{-}=\mathrm{MnOH}^{+}\left(\log \mathrm{K}_{1}^{\mathrm{OH}}=3.4\right) \\
{\left[\mathrm{MnOH}^{+}\right]=10^{\mathrm{pH}-10.6} \cdot\left[\mathrm{Mn}^{2+}\right]} \\
\mathrm{Mn}^{2+}+\mathrm{SO}_{4}^{2-}=\mathrm{MnSO}_{4}\left(\log \mathrm{K}_{1}=2.28\right) \\
{\left[\mathrm{MnSO}_{4}\right]=10^{2.28} \cdot\left[\mathrm{Mn}^{2+}\right]\left[\mathrm{SO}_{4}^{2-}\right]}
\end{gathered}
$$

and redox reactions:

$$
\begin{gathered}
\mathrm{MnO}_{4}^{-}+8 \mathrm{H}^{+}+5 \mathrm{e}^{-}=\mathrm{Mn}^{2+}+4 \mathrm{H}_{2} \mathrm{O}\left(\mathrm{E}_{0}=1.507 \mathrm{~V}\right) \\
{\left[\mathrm{MnO}_{4}^{-}\right]=\left[\mathrm{Mn}^{2+}\right] \cdot 10^{5 \cdot \mathrm{A} \cdot(\mathrm{E}-1.507)+8 \cdot \mathrm{pH}}} \\
\mathrm{MnO}_{4}^{-}+\mathrm{e}^{-}=\mathrm{MnO}_{4}^{2-}\left(\mathrm{E}_{0}=0.56 \mathrm{~V}\right) \\
{\left[\mathrm{MnO}_{4}^{-}\right]=\left[\mathrm{MnO}_{4}^{2-}\right] \cdot 10^{\mathrm{A} \cdot(\mathrm{E}-0.56)}} \\
\mathrm{Mn}^{3+}+\mathrm{e}^{-}=\mathrm{Mn}^{2+}\left(\mathrm{E}_{0}=1.509 \mathrm{~V}\right) \\
{\left[\mathrm{Mn}^{3+}\right]=\left[\mathrm{Mn}^{2+}\right] \cdot 10^{\mathrm{A} \cdot(\mathrm{E}-1.509)}} \\
2 \mathrm{H}_{2} \mathrm{CO}_{3}+2 \mathrm{H}^{+}+2 \mathrm{e}^{-}=\mathrm{H}_{2} \mathrm{C}_{2} \mathrm{O}_{4}+2 \mathrm{H}_{2} \mathrm{O}\left(\mathrm{E}_{0}=-0.386\right) \\
{\left[\mathrm{H}_{2} \mathrm{CO}_{3}\right]=\left[\mathrm{H}_{2} \mathrm{C}_{2} \mathrm{O}_{4}\right]^{0.5} \cdot 10^{\mathrm{A} \cdot(\mathrm{E}+0.396)+\mathrm{pH}}}
\end{gathered}
$$

where: $\mathrm{pH}=-\log \left[\mathrm{H}^{+}\right], \mathrm{A}=\mathrm{F} /(\mathrm{RT} \cdot \ln 10), \mathrm{F}=$ Faraday constant, $\mathrm{R}=$ gas constant, $\mathrm{T}=$ temperature $(\mathrm{K}) ; \mathrm{A}=$ 16.92 at $298 \mathrm{~K}$. All attainable (qualitative and quantitative) knowledge is involved in these balances and equilibrium constants. Other species, e.g. $\mathrm{Mn}\left(\mathrm{SO}_{4}\right)^{+3-2 i}$ complexes, are unknown in literature, see $[17,28]$. The requirement involved with independent set of equilibrium constants prevents receiving of contradictory equations.

It is assumed that the solution in the redox system is sufficiently acidified $\left(\mathrm{H}_{2} \mathrm{SO}_{4}\right)$ to prevent formation of $\mathrm{MnO}_{2}$, i.e., the system is homogeneous (solution) during the titration, and thus $\mathrm{MnO}_{2}$ is not involved in the balance (27). 


\section{Prevention of Contradictory Equations}

As were stated above, GEB is fully compatible with charge and concentration balances. The balances should be arranged rightly; it means that all known species from the system in question should be included properly and consequently in the related balances. All more complex species should be involved in expressions for the related equilibrium constants.

Tables of the equilibrium constants usually contain redundant and incompatible equilibrium constants values. The equilibrium constants were determined experimentally, then the quoted value of a defined equilibrium constant may be different in different tables. For example, the $\mathrm{pK} 1$ value found in for acetic acid is 4.65 [37] (at $\mathrm{I}=$ $0.1 \mathrm{~mol} / \mathrm{L}$, without indication of temperature) and 4.76 [38] (at $25^{\circ} \mathrm{C}$, without indication of ionic strength, I). However, some hidden divergence in the equilibrium data is usually encountered in the same book. For example, in [38] we have the following values of standard potentials: $\mathrm{E}_{01}=0.907 \mathrm{~V}$ for $\mathrm{Hg}^{2+}+2 \mathrm{e}^{-}=\mathrm{Hg}_{2}^{2+}, \mathrm{E}_{02}=$ $0.850 \mathrm{~V}$ for $\mathrm{Hg}^{2+}+2 \mathrm{e}^{-}=\mathrm{Hg}$, and $\mathrm{E}_{03}=0.792 \mathrm{~V}$ for $\mathrm{Hg}_{2}^{2+}+2 \mathrm{e}^{-}=2 \mathrm{Hg}$. Then we get $\mathrm{E}_{03},=2 \mathrm{E}_{02}-\mathrm{E}_{01}=$ $0.793 \neq \mathrm{E}_{03}$. Far greater discrepancies between equilibrium data at the apparent abundance of the physicochemical data may be quoted profusely. Referring to the systems presented in this paper, one can find $\mathrm{E}_{01}=1.69$ $\mathrm{V}$ for $\mathrm{MnO}_{4}^{-}+4 \mathrm{H}^{+}+3 \mathrm{e}^{-}=\mathrm{MnO}_{2}+2 \mathrm{H}_{2} \mathrm{O}, \mathrm{E}_{02}=2.26$ $\mathrm{V}$ for $\mathrm{MnO}_{4}^{2-}+4 \mathrm{H}^{+}+2 \mathrm{e}^{-}=\mathrm{MnO}_{2}+2 \mathrm{H}_{2} \mathrm{O}$, and $\mathrm{E}_{03}=$ $0.56 \mathrm{~V}$ for $\mathrm{MnO}_{4}^{-}+\mathrm{e}^{-}=\mathrm{MnO}_{4}^{2-}$; we state that $\mathrm{E}_{03},=\mathrm{E}_{02}$ $-\mathrm{E}_{01}=0.57 \neq \mathrm{E}_{03}$.

\section{Final Comments}

The properties of the linear combination $2 \cdot f(\mathrm{O})-f(\mathrm{H})$ of elemental balances: $f(\mathrm{H})$ for $\mathrm{H}$ and $f(\mathrm{O})$ for $\mathrm{O}$ were referred to non-redox and redox systems (aqueous media), denoted as System A and System B, respectively. It was assumed that the composition of titrand (D) is the same, but the titrants applied were different: $\mathrm{NaOH}$ in the System A, and $\mathrm{KMnO}_{4}$ in the System B.

In the System A (non-redox system), it was stated that $2 \cdot f(\mathrm{O})-f(\mathrm{H})$ is the linear combination of charge and other elemental or core balances: $f(\mathrm{Na}), f\left(\mathrm{SO}_{4}\right), f\left(\mathrm{C}_{2} \mathrm{O}_{4}\right)$ and $f\left(\mathrm{CO}_{3}\right)$, i.e. $2 \cdot f(\mathrm{O})-f(\mathrm{H})$ are not independent balances in this system.

In the System B (redox system) $2 \cdot f(\mathrm{O})-f(\mathrm{H})$ is independent on those balances. For redox systems, $2 \cdot f(\mathrm{O})-$ $f(\mathrm{H})$ is the primary form of Generalized Electron Balance (GEB), obtained according to Approach II.

These properties are valid for non-redox and redox systems of any degree of complexity. The linear dependency/independency property of $2 \cdot f(\mathrm{O})-f(\mathrm{H})$ is then a new criterion distinguishing non-redox and redox systems.
The Approach II is equivalent to the Approach I; the latter is based on the common pool of the electron principle. However, the Approach II offers special advantages, of capital importance, particularly when referred to redox systems, where complex organic species are considered. Namely, the knowledge of oxidation numbers of all elements and structure of the elements in these species is not needed. A known composition of all the species, expressed by their formula and external charge, provides information sufficient to formulate the related GEB. In particular, the Approach II is convenient in formulation of GEB for the systems where radicals and ion-radicals are formed. The Approach I to GEB, considered as a "short" version of GEB, is applicable for the systems where oxidation degree can easily be calculated for all elements in the redox system in question.

The properties of $2 \cdot f(\mathrm{O})-f(\mathrm{H})$ can be extended on non-redox or redox systems of any degree of complexity. These properties can also be extended on mixed-solvent systems, where polar protic (e.g. $\left.\mathrm{H}_{2} \mathrm{O}, \mathrm{CH}_{3} \mathrm{OH}\right)$ or aprotic (e.g. $\left.\left(\mathrm{CH}_{3}\right)_{2} \mathrm{SO}\right)$ solvents are involved.

The terms: "oxidant" and "reductant" are not necessary (not applicable) in considerations on a redox system [24-28]. Different species are considered in their natural form, i.e. as solvates, e.g. hydrates in aqueous media; although the systems with mixed solvents (e.g. binarysolvent systems) were also considered [40].

The Approach II shows that GEB is based on reliable principles of the matter conservation, and-in this regard - it is equally robust as equations for charge and concentration balances. From this viewpoint, GEB is considered as a relatively new law of Nature, referring to electrolytic redox systems of any degree of complexity, namely equilibrium, metastable, non-equilibrium, monoand poly-phase systems [28].

GEB completes the set of charge and concentration balances and a complete set of independent expressions for equilibrium constants, needed for quantitative description of redox system.

In summary, GEB, charge and concentration balances, together with the set of independent equilibrium constants, provide the numerical algorithm, implemented to software packages that support advanced programming, such as MATLAB computing environment. The calculation procedure, based on iterative computer programs [28], enables the desired relationships to be plotted graphically.

\section{REFERENCES}

[1] A. J. Bard and S. H. Simonsen, "The General Equation for the Equivalence Point Potential in Oxidation-Reduction Titrations," Journal of Chemical Education, Vol. 37, No. 7, 1960, pp. 364-366. http://dx.doi.org/10.1021/ed037p364 
[2] E. Bishop, "Some Theoretical Considerations in Analytical Chemistry. Part VI. The Precise Calculation of Data for Redox Titration Curves," Analytica Chimica Acta, Vol. 26, 1962, pp. 397-405.

http://dx.doi.org/10.1016/S0003-2670(00)88405-6

[3] J. A. Goldman, "The Equivalence Point Potential in Redox Titrations," Analytica Chimica Acta, Vol. 33, 1965, pp. 217-218. http://dx.doi.org/10.1016/S0003-2670(01)84877-7

[4] J. A. Goldman, "A General Equation for the Description of Redox Titration Curves," Journal of Electroanalytical Chemistry, Vol. 11, No. 4, 1966, pp. 255-261. http://dx.doi.org/10.1016/0022-0728(66)80090-6

[5] J. A. Goldman, "Further Considerations on Redox Titration Equations," Journal of Electroanalytical Chemistry, Vol. 11, No. 6, 1966, pp. 416-424. http://dx.doi.org/10.1016/0022-0728(66)80010-4

[6] J. A. Goldman, "The Locations of Inflection Points on Titration Curves for Symmetrical Redox Reactions," Journal of Electroanalytical Chemistry, Vol. 14, No. 4, 1967, pp. 373-383.

http://dx.doi.org/10.1016/0022-0728(67)80018-4

[7] J. A. Goldman, "Redox Equilibria. V. The Locations of Inflection Points on Titration Curves for Homogeneous Reactions," Journal of Electroanalytical Chemistry, Vol. 18, No. 1-2, 1968, pp. 41-45. http://dx.doi.org/10.1016/S0022-0728(68)80158-5

[8] J. A. Goldman, "Redox Equilibria. Part VI. General Titration Curve Equation for Homogeneous and Symmetrical Redox Reactions," Journal of Electroanalytical Chemistry, Vol. 19, No. 3, 1968, pp. 205-214. http://dx.doi.org/10.1016/S0022-0728(68)80119-6

[9] A. Meretoja, O. Lukkari and E. Hakoila, "Redox Titrations-II. Location of Inflection Points on Titration Curves for Homogeneous Redox Reactions," Talanta, Vol. 25, No. 10, 1978, pp. 557-562. http://dx.doi.org/10.1016/0039-9140(78)80146-5

[10] J. Stur, M. Bos and W. E. van der Linden, "A Generalized Approach for the Calculation and Automation of Potentiometric Titrations Part 2. Redox Titrations," Analytica Chimica Acta, Vol. 158, 1984, pp. 125-129. http://dx.doi.org/10.1016/S0003-2670(00)84819-9

[11] R. de Levie, "A Simple Expression for the Redox Titration Curve," Journal of Electroanalytical Chemistry, Vol. 323, No. 1-2, 1992, pp. 347-355. http://dx.doi.org/10.1016/0022-0728(92)80022-V

[12] R. de Levie, "Advanced Excel for Scientific Data Analysis," 2nd Edition, Oxford University Press, New York, 2008.

[13] G. Raj, "Advanced Physical Chemistry," 35th Edition, GOEL Publ. House, 2009.

[14] T. Michałowski, "Calculation of $\mathrm{pH}$ and Potential E for Bromine Aqueous Solutions," Journal of Chemical Education, Vol. 71, No. 7, 1994, pp. 560-562. http://dx.doi.org/10.1021/ed071p560

[15] T. Michałowski and A. Lesiak, "Acid-Base Titration Curves in Disproportionating Redox Systems," Journal of Chemical Education, Vol. 71, No. 8, 1994, pp. 632-636. http://dx.doi.org/10.1021/ed071p632
[16] T. Michałowski and A. Lesiak, "Formulation of Generalized Equations for Redox Titration Curves," Chemia Analityczna, Vol. 39, No. 4, 1994, pp. 623-637.

[17] T. Michałowski, N. Wajda and D. Janecki, "A Unified Quantitative Approach to Electrolytic Systems," Chemia Analityczna, Vol. 41, No. 4, 1996, pp. 667-685.

[18] T. Michałowski, "Calculations in Analytical Chemistry with Elements of Computer Programming," 2001. http://suw.biblos.pk.edu.pl/resourceDetails\&rId=3974

[19] T. Michałowski, A. Baterowicz, A. Madej and J. Kochana, "An Extended Gran Method and Its Applicability for Simultaneous Determination of Fe(II) and Fe(III)," Analytica Chimica Acta, Vol. 442, No. 2, 2001, pp. 287-293. http://dx.doi.org/10.1016/S0003-2670(01)01172-2

[20] T. Michałowski, M. Toporek and M. Rymanowski, "Overview on the Gran and Other Linearization Methods Applied in Titrimetric Analyses," Talanta, Vol. 65, No. 5, 2005, pp. 1241-1253.

http://dx.doi.org/10.1016/j.talanta.2004.08.053

[21] T. Michałowski, M. Rymanowski and A. Pietrzyk, "Nontypical Brønsted Acids and Bases," Journal of Chemical Education, Vol. 82, No. 3, 2005, pp. 470-472. http://dx.doi.org/10.1021/ed082p470

[22] T. Michałowski, K. Kupiec and M. Rymanowski, "Numerical Analysis of the Gran Methods: A Comparative Study," Analytica Chimica Acta, Vol. 606, No. 2, 2008, pp. 172-183. http://dx.doi.org/10.1016/j.aca.2007.11.020

[23] M. Ponikvar, T. Michałowski, K. Kupiec, S. Wybraniec and M. Rymanowski, "Experimental Verification of the Modified Gran Methods Applicable to Redox Systems," Analytica Chimica Acta, Vol. 628, No. 2, 2008, pp. 181189. http://dx.doi.org/10.1016/j.aca.2008.09.012

[24] T. Michałowski and A. Pietrzyk, "Complementarity of Physical and Chemical Laws of Conservation in Aspect of Electrolytic Systems (in Polish)," Wiadomości Chemiczne, Vol. 61, No. 7-8, 2007, pp. 625-640.

[25] http://www.chemia.uj.edu.pl/ ictchem/book.html

[26] T. Michałowski, "The Generalized Approach to Electrolytic Systems: I. Physicochemical and Analytical Implications," Critical Reviews in Analytical Chemistry, Vol. 40, No. 1, 2010, pp. 2-16. http://dx.doi.org/10.1080/10408340903001292

[27] T. Michałowski, A. Pietrzyk, M. Ponikvar-Svet and M. Rymanowski, "Critical Reviews in Analytical Chemistry," Vol. 40, No. 1, 2010, pp. 17-29. http://dx.doi.org/10.1080/10408340903001292

[28] T. Michałowski, "Application of GATES and MATLAB for Resolution of Equilibrium, Metastable and Non-Equilibrium Electrolytic Systems," Chapter 1, In: T. Michałowski, Ed., Applications of MATLAB in Science and Engineering, InTech, 2011, pp. 1-35.

http://www.intechopen.com/books/show/title/applications -of-matlab-in-science-and-engineering

[29] T. Michałowski, M. Ponikvar-Svet, A. G. Asuero and K. Kupiec, "Thermodynamic and Kinetic Effects Involved in the pH Titration of As(III) with Iodine in a Buffered Malonate System," Journal of Solution Chemistry, Vol. 41, No. 3, 2012, pp. 436-446. http://dx.doi.org/10.1007/s10953-012-9815-6 
[30] T. Michałowski, M. Toporek, A. M. Michałowska-Kaczmarczyk and A. G. Asuero, "New Trends in Studies on Electrolytic Redox Systems," Electrochimica Acta, Vol. 109, 2013, pp. 519-531.

[31] B. Nemzer, Z. Pietrzkowski, A. Spórna, P. Stalica, W. Thresher, T. Michałowski and S. Wybraniec, "Betalainic and Nutritional Profiles of Pigment-Enriched Red Beet Root (Beta vulgaris L.) Dried Extracts," Food Chemistry, Vol. 127, No. 1, 2011, pp. 42-53.

http://dx.doi.org/10.1016/i.foodchem.2010.12.081

[32] S. Wybraniec and T. Michałowski, "New Pathways of Betanidin and Betanin Enzymatic Oxidation," Journal of Agricultural and Food Chemistry, Vol. 59, No. 17, 2011, pp. 9612-9622. http://dx.doi.org/10.1021/jf2020107

[33] S. Wybraniec, P. Stalica, A. Spórna, B. Nemzer, Z. Pietrzkowski and T. Michałowski, "Antioxidant Activity of Betanidin: Electrochemical Study in Aqueous Media," Journal of Agricultural and Food Chemistry, Vol. 59, No. 22, 2011, pp. 12163-12170. http://dx.doi.org/10.1021/jf2024769

[34] S. Wybraniec, K. Starzak, A. Skopińska, M. Szaleniec, J. Słupski, K. Mitka, P. Kowalski and T. Michałowski, "Eff- ects of Metal Cations on Betanin Stability in AqueousOrganic Solutions," Food Science and Biotechnology, Vol. 22, No. 2, 2013, pp. 353-363. http://dx.doi.org/10.1007/s10068-013-0088-7

[35] S. Wybraniec, K. Starzak, A. Skopińska, B. Nemzer, Z. Pietrzkowski and T. Michalowski, "Studies on Non-Enzymatic Oxidation Mechanism in Neobetanin, Betanin and Decarboxylated Betanins, Journal of Agricultural and Food Chemistry, Vol. 61, No. 26, 2013, pp. 6465-6476.

[36] http://en.wikipedia.org/wiki/System_of_linear_equations

[37] J. Inczedy, “Analytical Applications of Complex Equilibria," Horwood, Chichester, 1976.

[38] Yu. Lurie, "Handbook of Analytical Chemistry," Mir Publishers, Moscow, 1975.

[39] B. P. Nikolsky, "Guide-Book for Chemist (in Russian)," Vol. 3, Khimia, Moscow, 1964.

[40] T. Michałowski, A. M. Michałowska-Kaczmarczyk and M. Toporek, "Formulation of General Criterion Distinguishing between Non-Redox and Redox Systems," Electrochimica Acta.

\section{Appendix (a More Sophisticated Presentation of the Linear Dependency)}

Let us take the set of linear equations:

$$
\begin{aligned}
& a_{11} x_{1}+a_{12} x_{2}+a_{13} x_{3}=b_{1} \\
& a_{21} x_{1}+a_{22} x_{2}+a_{23} x_{3}=b_{2}
\end{aligned}
$$

completed by linear combination of these equations, i.e.,

$$
c_{1}\left(a_{11} x_{1}+a_{12} x_{2}+a_{13} x_{3}\right)+c_{2}\left(a_{21} x_{1}+a_{22} x_{2}+a_{23} x_{3}\right)=\left(c_{1} a_{11}+c_{2} a_{21}\right) x_{1}+\left(c_{1} a_{12}+c_{2} a_{22}\right) x_{2}+\left(c_{1} a_{13}+c_{2} a_{23}\right) x_{3}=c_{1} b_{1}+c_{2} b_{2} \text {. }
$$

Applying matrix algebra we see that the determinant

$$
\boldsymbol{D}=\left|\begin{array}{ccc}
a_{11} & a_{12} & a_{13} \\
a_{21} & a_{22} & a_{23} \\
c_{1} a_{11}+c_{2} a_{21} & c_{1} a_{12}+c_{2} a_{22} & c_{1} a_{13}+c_{2} a_{23}
\end{array}\right|
$$

has zero value

$$
\boldsymbol{D}=c_{1} \cdot\left|\begin{array}{lll}
a_{11} & a_{12} & a_{13} \\
a_{21} & a_{22} & a_{23} \\
a_{11} & a_{12} & a_{13}
\end{array}\right|+c_{2} \cdot\left|\begin{array}{ccc}
a_{11} & a_{12} & a_{13} \\
a_{21} & a_{22} & a_{23} \\
a_{21} & a_{22} & a_{23}
\end{array}\right|=c_{1} \cdot 0+c_{2} \cdot 0=0
$$

irrespectively on the $c_{1}$ and $c_{2}$ values; at $\boldsymbol{D}=0$, calculation of $x_{1}, x_{2}$ and $x_{3}$ is impossible. On the other hand, even a small change in the third equation makes a system of equations contradictory. For example, taking the system of equations: (1a), (2a) (see text) and $(\alpha) x_{1}-7 x_{2}=-5.01,(\beta) 1.01 x_{1}-7 x_{2}=-5,(\gamma) x_{1}-7.01 x_{2}=-5$ instead of (3a) we get the contradictions: $-5 \neq-5.01$ in $(\alpha)$ and $(\gamma)$, and $-4.98 \neq-5$ in $(\beta)$. 